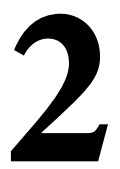

\title{
LA REPETICIÓN ESCOLAR EN EDUCACIÓN SECUNDARIA Y FACTORES DE RIESGO ASOCIADOS
}

(GRADE REPETITION IN SECONDARY EDUCATION AND ASSOCIATED RISK FACTOR)

Inmaculada Méndez Mateo

Fuensanta Cerezo Ramírez

Universidad de Murcia

DOI: $10.5944 / e d u c X X 1.20172$

Cómo referenciar este artículo/How to reference this article:

Méndez, I. y Cerezo, F. (2018). La repetición escolar en educación secundaria y factores de riesgo asociados. Educación XX1, 21(1), 41-62, doi: 10.5944/educXX1.20172

Méndez, I. \& Cerezo, F. (2018). La repetición escolar en educación secundaria y factores de riesgo asociados [Repetition rate in secondary education and associated risk factors]. Educación XX1, 21(1), 41-62, doi: 10.5944/educXX1.20172

\section{RESUMEN}

La alta tasa de alumnos repetidores es una de las preocupaciones del sistema educativo español. Los estudios revelan que el mayor porcentaje de repetidores se encuentra entre el alumnado del primer curso de Educación Secundaria Obligatoria, siendo más frecuente en los varones. La Educación Secundaria Obligatoria coindice con una etapa del ciclo vital de transformación que facilita conductas de riesgo en los adolescentes, entre ellas, la repetición de curso, el consumo de drogas y determinadas conductas agresivas como el acoso escolar.

Este estudio tiene por objeto examinar cuáles son los principales factores relacionados con la repetición de curso atendiendo al estudio de características sociodemográficas (ámbito, titularidad, sexo, procedencia y edad), dinámica bullying (agresor o víctima); factores de riesgo/ protección para el consumo de drogas (familia, amigos y ámbito escolar), el grado de adaptación/inadaptación general (personal, social y escolar), la insatisfacción con el ambiente familiar e insatisfacción con los hermanos. Los participantes, 1267 
estudiantes, de entre 11 y 18 años $(M=14.41 ; D t=1.427)$, siendo $49.3 \%$ varones, cumplimentaron el Test de Evaluación sociométrica de la violencia entre escolares Bull-S; el instrumento FRIDA -Factores de Riesgo Interpersonales para el consumo de Drogas en Adolescentes- y el Test Autoevaluativo Multifactorial de Adaptación infantil -TAMAI-.

Los resultados indican que la repetición de curso se ve relacionada con variables como: titularidad del centro educativo, sexo, procedencia, edad; el rol de agresor en acoso escolar; accesibilidad, factores de riesgo familiares (reacción familiar ante el consumo, riesgo familiar, actividades protectoras) para el consumo de drogas así como inadaptación escolar e insatisfacción con el ambiente familiar. Estos resultados evidencian la necesidad de llevar a cabo acciones de cooperación entre las familias y los centros educativos, así como poner a disposición del profesorado recursos que permitan detectar y abordar situaciones de riesgo y prevenir la repetición de curso.

\section{PALABRAS CLAVE}

Acoso escolar; adolescencia; abuso de drogas; tasa de repetición de curso.

\section{ABSTRACT}

The high rate of pupils that repeat a grade at school is a concern for the Spanish educational system. Studies show that the highest percentage of repeaters is among students of the first grade of secondary education, with a higher frequency of males. Secondary School coincides with a stage in the life cycle of transformation, facilitating risk behaviors among adolescents, including grade repetition, drug use and certain aggressive behaviors such as bullying.

This study aims to examine the main factors associated with repetition, taking into consideration the socio-demographic characteristics (scope, ownership, gender, origin and age), bullying (bully or victim); risk or protective factors for drug use (family, friends or school context), level of general adjustment/maladjustment (personal, social and scholar); dissatisfaction with the home environment and with siblings. The participants, 1,267 students aged from 11 to $18(\mathrm{M}=14.41$; $\mathrm{Dt}=1.427)$, 49.3\% males, completed the sociometric test evaluation of violence among school mates Bull-S, the FRIDA questionnaire (Interpersonal Risk Factors for Adolescent Drug Consumption) and the Self-reported Multifactor Child Adaptation Test -TAMAI-.

The results indicate that repeating a grade in school is related to variables such as: title of the school, sex, origin, age, the role of the aggressor in bullying, accessibility, family risk factors (family reaction to drug use, family risk, protection activities) for drug consumption as well as maladjustment 
to school and dissatisfaction with the family environment. These results demonstrate the need to carry out cooperation actions between families and schools and to make resources available that allow teachers to identify and address risk and prevent repetition.

\section{KEY WORDS}

Bullying; teen agers; drug abuse; repetition rate.

\section{INTRODUCCIÓN}

Según los datos del Instituto Nacional de Evaluación Educativa (2016) es destacable que el mayor porcentaje del alumnado que ha sido repetidor en nuestro país se produce en el primer curso de Educación Secundaria Obligatoria, presentando una tendencia a disminuir a medida que va aumentando el curso (repite el 12.6\% del primer curso frente al $8.6 \%$ de cuarto curso) teniendo mayor incidencia en los centros de titularidad pública. Siendo más frecuente entre los varones en todos los cursos, presentando un porcentaje de repetición superior al de las chicas en 4.3 puntos, especialmente en primer curso. Estos datos vienen a coincidir con lo apuntado por Lozano Díaz (2009) cuando afirma que las mujeres rinden mejor que los varones y que el número de alumnos repetidores aumenta con la edad, lo que viene a determinar que no sigan el aprendizaje del curso para su edad.

A este hecho cabe destacar que la alta tasa de alumnos repetidores existente en nuestro país (OCDE, 2016), superior a la media de la Unión Europea (el 34\% ha repetido un curso frente al 16\% del conjunto de países de la OCDE), es una de las mayores preocupaciones del Ministerio de Educación, Cultura y Deporte en España, ya que la repetición de curso está vinculada con el fracaso escolar además de con otras situaciones de riesgo para el desarrollo social y personal. Entre los factores que inciden en esta situación se encuentra el alumnado inmigrante, la inasistencia a cursos de preescolar, la estructura familiar así como la ausencia de libros en el hogar (Cordero, Manchón y Simancas, 2014).

La repetición de curso, no es solo una cuestión académica, de hecho, como señalan Farías, Fiol, Kit y Melgar (2007) el impacto de la repetición en el alumnado indica sobreedad en su curso, lo que está asociado con resultados negativos, la estigmatización de los pares y otras experiencias que pueden exacerbar problemas de conducta y de ajustes socioemocionales. 
En el rendimiento académico de los menores influye activamente la implicación de los progenitores. Suárez et al. (2011), tras la revisión de diversos estudios, encontraron que en la mayoría de los casos, la implicación de los progenitores en la educación de los hijos ejerce efectos positivos, siendo, por tanto, un factor a tener en cuenta cuando se analiza la repetición de curso.

Por otra parte, la transición a la Educación Secundaria Obligatoria coincide con una etapa del ciclo vital de transformación psicológica, biológica y emocional, que puede manifestarse como falta de motivación e implicación en el estudio, lo que en ocasiones puede llevar a la repetición de curso, al fracaso escolar y por ende aumentar el riesgo de abandono del sistema educativo, cerrando la posibilidad de alcanzar el éxito académico (Martínez González y Álvarez Blanco, 2005).

En la adolescencia se hace imprescindible la necesidad de pertenecer a un grupo, ser aceptados y sentirse reflejados en una cohorte de iguales (Sullivan, K., Cleary y Sullivan, G. 2005). García, Sureda y Monjas (2010) aluden a que el rechazo entre iguales es un proceso interpersonal que acarrea consecuencias negativas para los alumnos rechazados. De manera que, el grupo de iguales puede proteger al sujeto ante las amenazas externas o puede ejercer una influencia negativa hacia la implicación en actos delictivos (Garaigordobil y Oñederra, 2010). Así, algunos estudios señalan que el consumo de drogas se ve favorecido por la existencia de modelos consumidores en el grupo de amigos, cuando entre ellos existe una actitud y comportamiento favorable hacia el mismo (Alfonso, Huedo-Medina y Espada, 2009) facilitando el acceso a las drogas (Cerezo, Méndez y Ato, 2013) y sobre todo por la influencia de la figura del mejor amigo (Espada, Pereira y García-Fernández, 2008).

El consumo de drogas está determinado por diversos factores de naturaleza multidimensional (Carballo et al., 2004). Entre los factores escolares, los estudios destacan: la insatisfacción escolar, bajo nivel de compromiso en las actividades académicas, absentismo escolar unido a bajo rendimiento académico, repetición de curso, lo que ocasiona fracaso escolar y conductas problemáticas en el aula (Palacios y Andrade, 2007; Pinazo, Pons y Carreras, 2002). Entre los factores de riesgo familiar destacan el clima de insatisfacción respecto al sistema familiar, estrategias educativas donde se utiliza el castigo (Pons, 1998), reacción de indiferencia ante el consumo de drogas (Cerezo et al., 2013); el modelado familiar (Inglés et al., 2007); consumo en el ámbito familiar (Pons, 1998), sobre todo cuando el hermano así como el progenitor es consumidor (Espada et al. 2008; Ruiz-Juan y RuizRisueño, 2011). 
Según la Delegación del Gobierno para el Plan Nacional sobre DrogasDGPNSD- (2016), el consumo de alcohol aumenta entre los 14 y 15 años así como la frecuencia de mujeres consumidoras entre los 14 y 16 años. Se destaca la facilidad de acceso al alcohol por parte de los menores unido a una baja percepción de riesgo.

De otra parte, los problemas de relación entre los iguales, origen del acoso escolar (Garaigordobil y Oñederra, 2010), plantea la necesidad de conocer el estatus social de cada miembro del grupo, esto es, su situación sociométrica (líder, popular, promedio, aislado, rechazado (Cerezo, 2012).

Según Olweus (1998) para que un estudiante se convierta en víctima del bullying ha de estar expuesto de forma repetida e intencionalmente a acciones negativas que le ocasionan daño, le hieren o le incomodan, que son llevadas a cabo por otro estudiante o varios de ellos, existiendo un desequilibrio de fuerzas, dícese de una relación de poder asimétrica, siendo la víctima incapaz de defenderse; Cerezo (2009) ratifica el carácter intencional, perjudicial y persistente de este fenómeno. El acoso escolar, por tanto, constituye una dinámica asidua de exclusión, violencia e incluso deterioro de la socialización, de un estudiante o grupo de estudiantes, hacia otro compañero al que convierte en su víctima habitual.

Se encuentran relaciones significativas entre los roles de los implicados en acoso escolar y rendimiento académico. Diversos estudios aluden que en el agresor aparecen los siguientes efectos a medio y largo plazo: bajo rendimiento académico y fracaso escolar; rechazo al centro educativo; inadaptación escolar; no sigue el ritmo de aprendizaje del grupo-aula; repetición de curso, etc. (Cerezo, 1998; Pelegrín y Garcés de Los Fayos, 2009). Mientras que, el alumno víctima presenta un rendimiento académico superior al de los alumnos agresores (Cerezo, 2001).

Entre las formas de maltrato más habituales los estudios realizados destacan las siguientes: insultos y las amenazas, seguidos de maltrato físico, la exclusión social y otras formas (Cerezo, 2009; Cerezo y Méndez, 2012). Álvarez - García, Dobarro, Álvarez, Núñez y Rodríguez (2014) aluden a otros tipos de violencia escolar más graves como las amenazas con navajas u otros objetos y la violencia a través de las TIC como el cyberbullying que está en aumento (Hymel y Swearer (2015). En cuanto a la frecuencia de ocurrencia, suele producirse con una frecuencia de «una o dos veces por semana»o «rara vez», seguido de «todos los días» y «nunca» (Cerezo y Méndez, 2012). En la etapa de educación secundaria, los datos resaltan que los lugares dentro del ámbito escolar en los cuales suele ocurrir el fenómeno destacan: los espacios-aulas, seguidos del patio de recreo y los lugares de acceso al centro educativo (pasillos) y otros lugares (Cerezo, 2009; Cerezo y Méndez, 2012). 
Por otra parte, se deben tener en cuenta otros factores, como recoge la Teoría para la conducta de riesgo de los adolescentes (Jessor et al., 2003): factores de riesgo y de protección interrelacionados entre sí, como los relativos al carácter biológico-genéticos, al medio social (pobreza, desigualdad cultural, etc.), al medio percibido (modelos de conducta desviada y conflictos normativos entre padres y amigos, etc.), conductuales (bajo rendimiento escolar, etc.) y de personalidad. Dichos factores incitarán a unas conductas de riesgo, conformando un estilo de vida del adolescente que podrá caracterizarse por la presencia de conductas problema como el uso de drogas, el fracaso escolar y el abandono escolar. Por lo tanto, las conductas de riesgo o conductas problema se han de considerar conjuntamente, puesto que todos los elementos que componen esta teoría se encuentran en continua interacción causal, recíproca y bidireccional.

Tras lo expuesto, cabe citar que la repetición escolar ha sido estudiada como una conducta de riesgo de forma independiente o relacionada con otras conductas de riesgo como el consumo de drogas. A este respecto, Carabaña (2013) en su análisis llevado a cabo sobre los determinantes de la repetición escolar indica la existencia de varias causas. Cordero et al. (2014) mencionan que entre los factores condicionantes de la repetición escolar se encuentran los individuales y escolares, que incluso trascienden a la propia escuela y señalan una perspectiva internacional, es decir, factores relacionados con la política educativa y el contexto nacional dentro de un conjunto de países.

Así pues, se hace necesario avanzar en el análisis sobre los principales factores relacionados con la repetición escolar desde una perspectiva ecológica: individuo, grupo familiar, grupo escolar y aspectos sociodemográficos. Es por ello que este trabajo se plantea ahondar en el conocimiento de los factores de riesgo asociados a la repetición escolar desde esta perspectiva. Este objetivo general, se concreta en establecer la asociación entre la repetición escolar y los siguientes aspectos: a) características sociodemográficas (ámbito del centro educativo, titularidad del centro educativo, sexo, edad, procedencia); b) acoso escolar (agresor, víctima, estatus, impacto social, preferencia social; elementos situacionales como: formas, frecuencia y lugares de las agresiones así como la percepción de su gravedad y atribución de seguridad en el centro escolar); c) factores de riesgo/ protección para el consumo de drogas; d) el grado de adaptación/inadaptación personal, social y escolar así como el grado de insatisfacción con el ambiente familiar y con los hermanos. 


\section{MÉTODO}

\section{Participantes}

Los participantes del estudio fueron 1267 estudiantes de los 1509 que había previamente a los criterios de inclusión y exclusión (detallados en el procedimiento), de diferentes centros de Educación Secundaria públicos, privados concertados y privados de la Región de Murcia. La edad osciló entre los 11 y 18 años de edad ( $M=14.41 ; D \mathrm{t}=1.427)$, siendo $49.3 \%$ varones. El $83.7 \%$ eran de procedencia española y el $16.3 \%$ de extranjeros.

Los estudiantes pertenecían a los siguientes cursos de Educación Secundaria Obligatoria: 354 (27.9\%) de primer curso; 204 (16.1\%) de segundo curso; $299(23.6 \%)$ de tercer curso y $410(32.4 \%)$ de cuarto curso.

En cuanto a la repetición de curso, el 35.4\% habían repetido curso alguna vez.

\section{Instrumentos}

Para conocer la posición sociométrica de los participantes y su rol en bullying se utilizó el Test Bull-S Test de Evaluación de la Agresividad entre escolares Bull-S en su versión 3.3 (Cerezo, 2012), en su forma A (Alumnado). Se trata de un instrumento compuesto por 15 ítems de elección directa y tipo Likert para la medida de la agresividad entre iguales en contextos escolares, dirigido a todos los sujetos que integran el grupo-aula. Consta de tres dimensiones:

- Dimensión 1. Posición sociométrica. Mediante 4 ítems de nominación directa, analiza la situación sociométrica: estatus social de cada miembro en el grupo como variable nominal; impacto social, preferencia social como variables cuantitativas.

- Dimensión 2. Dinámica bullying. Formada por 6 ítems de nominación directa detecta a los estudiantes implicados en la dinámica bullying en sus distintos roles (agresor y víctima como variables cuantitativas).

- Dimensión 3. Percepción situacional. Emplea 5 ítems para analizar los aspectos situacionales en las relaciones de agresividad entre iguales: forma y lugares (variable nominales), frecuencia y cómo se perciben las situaciones de agresividad (nivel de gravedad atribuida y topografía así como nivel de seguridad percibida en el centro 
educativo) siendo estas últimas variables ordinales y que requieren una forma de respuesta de tipo Likert con 4 puntos de respuesta o bien mediante la elección de una respuesta entre cuatro posibles.

El cuestionario también recoge variables sociodemográficas: sexo, edad, procedencia, repetición de curso, titularidad y ubicación geográfica del centro educativo.

En su versión revisada demuestra ser un instrumento fiable (Alfa de Cronbach para Agresor =.734; Alfa de Cronbach para Víctima =.837) y válido para la evaluación del bullying. En los sujetos del presente estudio mostró valores similares (Alfa de Cronbach para Agresor =.83; Alfa de Cronbach para Víctima =.84).

Para evaluar los factores de riesgo para el consumo se administró el instrumento FRIDA -Factores de Riesgo Interpersonales para el consumo de Drogas en Adolescentes- (Secades, Carballo, Fernández, García Rodríguez y García Cueto, 2006). Consta de 90 ítems tipo Likert y analiza los siguientes siete factores: reacción de la familia ante el consumo (formado por los ítems del 1 al 15), grupo de amigos (ítems del 16 al 27), acceso a las drogas (ítems del 28 al 35), riesgo familiar (ítems del 36 al 51), educación familiar en drogas (ítems del 52 al 58), actividades protectoras (ítems del 59 al 81) y estilo educativo (ítems del 82 al 90). Las puntuaciones directas obtenidas en cada factor (variables continuas) son transformadas en una escala que oscila del 1 al 20 (Secades et al., 2006). El cuestionario posee un índice de fiabilidad alfa de Cronbach de.925 para el conjunto del mismo (Secades et at., 2006). En nuestro estudio fue de.928.

La evaluación de la adaptación/ inadaptación se llevó a cabo mediante el Test Autoevaluativo Multifactorial de Adaptación infantil -TAMAI- (Hernández- Guanir, 2015). Consta de 175 ítems de respuesta dicotómica que evalúa actitudes y comportamientos respecto a sí mismo (área personal), la relación social, ámbito escolar, ámbito familiar y las relaciones con los hermanos. Está formado por los siguientes factores: inadaptación personal (se obtiene sumando el número de elementos afirmativos del 1 al 22 y los elementos negativos del 61 a 77), inadaptación escolar (suma de los elementos afirmativos de los ítems 23 a 42 y los negativos de 78 a 88), inadaptación social (suma de elementos afirmativos del 43 a 60 y los negativos del 89 a 105), inadaptación general (suma de los valores finales de la inadaptación personal, escolar y social), insatisfacción con el ambiente familiar (contabilizando los elementos afirmativos de los ítems 106 a 110) e insatisfacción con los hermanos (sumando los elementos afirmativos de los números 111 a 115). Las puntuaciones directas de cada factor (variables continuas) son transformadas en puntuacio- 
nes normalizadas atendiendo a los baremos en población española según el sexo y el nivel educativo (segundo nivel para $1 .^{\circ}$ y $2 .^{\circ}$ curso de ESO y el tercer nivel para $3 .^{\circ}$ y $4 .^{\circ}$ curso de ESO). La escala ha demostrado su utilidad en un amplio rango de niveles educativos (desde primaria a bachillerato). La prueba posee un índice de fiabilidad alfa de Cronbach de .92. Para los sujetos del presente estudio, se obtuvo un coeficiente alfa de Cronbach de .944.

\section{Procedimiento}

Previamente se solicitó la autorización a la Consejería de Educación, Formación y Empleo de la Región de Murcia. A continuación, se seleccionaron los centros de enseñanza secundaria de manera representativa de la población de estudiantes matriculados (cursos académicos 2008-2009 y 2009-2010). Se realizó una entrevista con los directores y/u orientadores de los centros educativos seleccionados para exponer los objetivos del estudio y los instrumentos de evaluación. Tras solicitar el permiso del centro y promover su colaboración, se informó al Consejo Escolar pertinente para tramitar la autorización de los padres, madres o representantes legales de los estudiantes. En cada centro educativo se confeccionó un calendario de trabajo y se seleccionaron aleatoriamente los grupos. En la fase de administración de los instrumentos de medida, estaban presentes además de los encuestadores los profesores/ tutores durante dos sesiones de 50 minutos, preferentemente en horario de tutorías. Los estudiantes fueron informados de la confidencialidad y anonimato de los datos y sobre los objetivos del estudio. La participación fue voluntaria.

Los criterios de inclusión utilizados fueron los siguientes: estudiantes matriculados en un centro educativo perteneciente a la provincia de Murcia en los niveles de primer y segundo ciclo de Enseñanza Secundaria Obligatoria; obtener la autorización pertinente de padres, madres o representantes legales y que asistiesen a clase en las sesiones de administración de los instrumentos de medida. Del mismo modo, se tuvieron en cuenta los siguientes criterios de exclusión en el estudio: que por motivos de idioma no hubiese posibilidad de que comprendiesen los instrumentos de evaluación; que por alguna discapacidad no fuese posible cumplimentar los cuestionarios de evaluación; que no hubiesen asistido a clase o que hubiesen sido expulsados del centro educativo coincidiendo con las sesiones de administración de los instrumentos de evaluación y que no se hubiese cumplimentado alguno de los instrumentos de medida. 


\section{Análisis}

Para el estudio se ha seguido un diseño no experimental de tipo transversal. Se llevó a cabo el tratamiento estadístico de los datos a través del paquete estadístico SPSS -Statistical Package for the Social Sciences(versión 19.0). Tras comprobar los supuestos paramétricos, se utilizaron: técnicas descriptivas (frecuencias, porcentajes, media y desviación típica), tablas de contingencia con la prueba Chi-Cuadrado de Pearson para analizar variables categóricas como las variables sociodemográficas y la repetición escolar (haber repetido curso o no) como: titularidad del centro educativo (público/privada), sexo (hombre/mujer) así como ubicación geográfica (urbana/rural), estatus sociométrico (líder, popular, muy rechazado, rechazado, aislado, controvertido y promedio), tipo de agresiones (insultos y amenazas, maltrato físico, rechazo y otras), lugar de las agresiones (aula, patio, pasillos y otros), frecuencia de las agresiones (nunca, 1-2 veces por semana, rara vez y todos los días), gravedad de las agresiones (poco o nada, regular, bastante y mucho), seguridad en el centro (poco o nada, regular, bastante y mucho). Para cada tabla de contingencia se comprobó si cumplían la condición de validez, utilizando la corrección de Yates, en el caso de que el valor de las frecuencias esperadas fuese inferior o igual a 5 en tablas de contingencia $2 \times 2$. Del mismo modo, para determinar el tamaño del efecto se utilizó el Coeficiente Phi cuando se trataba de tablas de contingencia 2 x 2 o el coeficiente V de Cramer en el resto de tablas de la prueba Chi-Cuadrado de Pearson. Se utilizó el análisis de diferencias de medias a través de la prueba t de Student para muestras independientes utilizando como variable categórica dicotómica la repetición de curso y como variables dependientes continuas las siguientes: edad, agresivo, victima, impacto social, preferencia social, reacción de la familia ante el consumo, grupo de amigos, acceso a las drogas, riesgo familiar, educación familiar en drogas, actividades protectoras y estilo educativo; inadaptación personal, inadaptación escolar, inadaptación social, inadaptación general, insatisfacción con el ambiente familiar e insatisfacción con los hermanos. Se llevó a cabo el análisis del tamaño del efecto de Cohen calculando la diferencia de medias estandarizada expresada en valor absoluto (d).

\section{Resultados}

Variables sociodemográficas y repetición de curso

La prueba Chi-cuadrado de Pearson, indicaba que la distribución de la muestra presentaba una asociación significativa entre repetición de curso y las siguientes variables: Titularidad del Centro Educativo $(V=.215 ; \mathrm{p}=.000)$; 
Sexo $(V=.069 ; \mathrm{p}=.014)$ y Procedencia en la prueba Chi-cuadrado corregida de Yates $(\Phi=-.217, \mathrm{p}=.000)$.

En cuanto a la edad, la prueba t de Student mostró diferencias de medias significativas, asumiendo varianzas heterogéneas, en repetición de curso $T(877.606)=15.494 ; \mathrm{p}=.000$ con un efecto alto $(d=.91)$. A este respecto, la media de los repetidores fue superior $(M=15.19 ; D T=1.36)$ a la media de los no repetidores $(M=13.99 ; D T=1.28)$.

La ubicación geográfica del centro educativo en zona rural o urbana no mostró diferencias de medias significativas en la prueba Chi-cuadrado de Pearson (véase Tabla 1 con la prueba Chi-cuadrado de Pearson y su significación estadística, tabla de contingencia entre alumnos repetidores y no repetidores para cada una de las variables sociodemográficas).

Tabla 1

Variables sociodemográficas según la repetición de curso

\begin{tabular}{|c|c|c|c|c|c|}
\hline \multicolumn{2}{|c|}{$\begin{array}{c}\text { Variables } \\
\text { Sociodemográficas }\end{array}$} & $\begin{array}{c}\text { No repetidor } \\
\mathbf{n}=818 \\
(100 \%)\end{array}$ & $\begin{array}{c}\text { Repetidor } \\
\mathrm{n}=449 \\
(100 \%)\end{array}$ & $\chi^{2}$ & $\mathbf{p}$ \\
\hline \multirow{2}{*}{$\begin{array}{l}\text { Ámbito } \\
\text { geográfico }\end{array}$} & Urbano & $607(74.2 \%)$ & $315(70.2 \%)$ & 2.399 & n.s. \\
\hline & Rural & $211(25.8 \%)$ & $134(29.8 \%)$ & & \\
\hline \multirow[t]{2}{*}{ Titularidad } & Público & $480(41.3 \%)$ & $359(80 \%)$ & 58.660 & .000 \\
\hline & Privado & $338(58.7 \%)$ & $90(20 \%)$ & & \\
\hline Sexo & $\begin{array}{l}\text { Hombre } \\
\text { Mujer }\end{array}$ & $\begin{array}{l}382(46.7 \%) \\
436(53.3 \%)\end{array}$ & $\begin{array}{l}242(53.9 \%) \\
207(46.1 \%)\end{array}$ & 6.010 & .014 \\
\hline Procedencia & $\begin{array}{l}\text { Español } \\
\text { Extranjero }\end{array}$ & $\begin{array}{r}733(89.6 \%) \\
85(10.4 \%)\end{array}$ & $\begin{array}{l}327(72.8 \%) \\
122(27.2 \%)\end{array}$ & 59.717 & .000 \\
\hline
\end{tabular}

Nota: n.s.: no significativo

Problemática bullying y repetición de curso

Mediante el análisis de diferencias de medias con la prueba t de Student, asumiendo varianzas heterogéneas, se encontró que la variable repetición de curso mostraba diferencias de medias significativas con respecto a ser agresivo en acoso escolar $T(698.981)=5.243 ; \mathrm{p}=.000$, con un tamaño del efecto bajo $(d=.31)$; pero no entre las víctimas (véase en la Tabla 2 las medias y desviaciones típicas de la prueba t de Student para los alumnos 
repetidores y no repetidores en cada una de las variables estudiadas de la problemática bullying). También se hallaron diferencias significativas en Preferencia social $T(905.863)=-4.771 ; \mathrm{p}=.000$ con un tamaño del efecto bajo $(d=-.28)$. Sin embargo no aparecían en Impacto Social.

Tabla 2

Problemática bullying en función de la repetición de curso

\begin{tabular}{cccc}
\hline & & Repetidor & No repetidor \\
\hline \multirow{2}{*}{ Agresivo } & $M$ & 10.04 & 5.45 \\
& $D . T$. & $(16.46)$ & $(11.64)$ \\
\hline \multirow{2}{*}{ Víctima } & $M$ & 8.47 & 8.09 \\
& $D . T$. & $(15.60)$ & $(14.21)$ \\
\hline \multirow{2}{*}{ Impacto social } & $M$ & 9.87 & 9.87 \\
& $D . T$. & $(6.22)$ & $(6.55)$ \\
\hline \multirow{2}{*}{ Preferencia social } & $M$ & -.64 & 1.69 \\
& $D . T$. & $(8.36)$ & $(8.19)$ \\
\hline
\end{tabular}

En el análisis de la distribución mediante la prueba Chi-cuadrado de Pearson, se encontró una asociación significativa entre repetición de curso y Estatus $(V=.180 ; \mathrm{p}=.000)$, siendo el más común entre los repetidores (véase en la Tabla 3 la prueba Chi-cuadrado de Pearson y su significación estadística, tabla de contingencia entre alumnos repetidores y no repetidores para la variable estatus, las variables situacionales y percepción de seguridad y gravedad en el centro educativo).

En el caso de las variables situacionales, la prueba Chi-cuadrado de Pearson mostró la existencia de una asociación significativa atendiendo al tipo de agresiones $(V=.100 ; \mathrm{p}=.004)$ y lugar en el que ocurrían las agresiones $(V=.099 ; \mathrm{p}=.004)$, pero no en cuanto a la frecuencia de ocurrencia de las agresiones.

Con respecto a la percepción atribuida a las situaciones de acoso escolar, se encontró una asociación estadísticamente significativa entre la percepción de seguridad/inseguridad en el centro escolar, en función de la repetición de curso de los estudiantes $(V=.144, \mathrm{p}=.000)$; sin embargo no se encontraron diferencias en gravedad atribuida a las agresiones según la repetición de curso. 
Tabla 3

Estatus, variables situaciones y percepción de seguridad y gravedad en el centro en función de la repetición de curso

\begin{tabular}{|c|c|c|c|c|c|}
\hline \multicolumn{2}{|c|}{$\begin{array}{c}\text { Estatus, variables situacionales } \\
\text { y percepción de seguridad } \\
\text { y gravedad en el centro }\end{array}$} & \multirow{2}{*}{$\begin{array}{c}\text { No repetidor } \\
22(2.7 \%)\end{array}$} & \multirow{2}{*}{$\begin{array}{c}\text { Repetidor } \\
5(1.1 \%)\end{array}$} & \multirow{2}{*}{$\begin{array}{c}\chi^{2} \\
41.259\end{array}$} & \multirow{2}{*}{$\begin{array}{c}\mathbf{p} \\
.000\end{array}$} \\
\hline Estatus & Líder Popular & & & & \\
\hline & & $82(10 \%)$ & $17(3.8 \%)$ & & \\
\hline & Muy rechazado & $23(2.8 \%)$ & $17(3.8 \%)$ & & \\
\hline & Rechazado & $28(3.4 \%)$ & $43(9.6 \%)$ & & \\
\hline & Aislado & $118(14.4 \%)$ & $58(12.9 \%)$ & & \\
\hline & Controvertido & $4(0.5 \%)$ & 0 & & \\
\hline & Promedio & $541(66.1 \%)$ & $309(68.8 \%)$ & & \\
\hline \multirow[t]{4}{*}{ Tipo de agresiones } & Insultos y amenazas & $364(51.4 \%)$ & $205(52.6 \%)$ & 13.371 & .004 \\
\hline & Maltrato físico & $34(4.8 \%)$ & $38(9.7 \%)$ & & \\
\hline & Rechazo & $286(40.4 \%)$ & $130(33.3 \%)$ & & \\
\hline & Otras & $24(3.4 \%)$ & $17(4.4 \%)$ & & \\
\hline \multirow[t]{4}{*}{ Lugar agresiones } & Aula & $322(46.5 \%)$ & $198(50.9 \%)$ & 10.680 & .014 \\
\hline & Patio & $254(36.7 \%)$ & $110(28.3 \%)$ & & \\
\hline & Pasillos & $64(9.2 \%)$ & $53(13.6 \%)$ & & \\
\hline & Otros & $52(7.5 \%)$ & $28(7.2 \%)$ & & \\
\hline \multirow{4}{*}{$\begin{array}{l}\text { Frecuencia de las } \\
\text { agresiones }\end{array}$} & Nunca & $69(9 \%)$ & $44(10.7 \%)$ & 3.078 & n.s. \\
\hline & $1-2$ veces por semana & $358(46.7 \%)$ & $172(41.6 \%)$ & & \\
\hline & Rara vez & $219(28.6 \%)$ & $125(30.3 \%)$ & & \\
\hline & Todos los días & $120(15.7 \%)$ & $72(17.4 \%)$ & & \\
\hline \multirow{4}{*}{$\begin{array}{l}\text { Gravedad de las } \\
\text { agresiones }\end{array}$} & Poco o nada & $241(31.5 \%)$ & $132(31.8 \%)$ & 3.610 & n.s. \\
\hline & Regular & $286(37.3 \%)$ & $144(34.7 \%)$ & & \\
\hline & Bastante & $172(22.5 \%)$ & $89(21.4 \%)$ & & \\
\hline & Mucho & $67(8.7 \%)$ & $50(12 \%)$ & & \\
\hline \multirow{4}{*}{$\begin{array}{l}\text { Seguridad en el } \\
\text { centro }\end{array}$} & Poco o nada & $21(2.7 \%)$ & $35(8.5 \%)$ & 24.496 & .000 \\
\hline & Regular & $118(15.3 \%)$ & $68(16.4 \%)$ & & \\
\hline & Bastante & $335(43.4 \%)$ & $143(34.5 \%)$ & & \\
\hline & Mucho & $298(38.6 \%)$ & $168(40.6 \%)$ & & \\
\hline
\end{tabular}


Factores de riesgo para el consumo de drogas y repetición de curso

La prueba t de Student mostró diferencias de medias significativas, asumiendo varianzas heterogéneas en la reacción de la familia ante el consumo de drogas, $T(715.398)=2.636 ; \mathrm{p}=.009$ con un efecto bajo $(d=.15)$. La media de los repetidores fue superior a la media de los no repetidores (véase Tabla 4 las medias y desviaciones típicas de la prueba t de Student para los alumnos repetidores y no repetidores en cada uno de los factores de riesgo o de protección para el consumo de drogas).

En el acceso a las drogas la prueba t de Student mostró diferencias de medias significativas, asumiendo varianzas heterogéneas, en repetición de curso $T$ (814.192)=-3.706; $\mathrm{p}=.000$ con efecto bajo $(d=-.22)$. La media de los no repetidores fue superior a la media de los repetidores.

En riesgo familiar para el consumo de drogas la prueba t de Student mostraba diferencias de medias significativas, asumiendo varianzas heterogéneas, en repetición de curso $T$ (720.703)=-3.102; $\mathrm{p}=.002$ con efecto bajo $(d=-.18)$. La media de los no repetidores fue superior a la media de los repetidores.

Asimismo, las actividades protectoras frente al consumo de drogas, mostraba diferencias de medias significativas, asumiendo varianzas heterogéneas, en repetición de curso $T(650.859)=-4.010 ; \mathrm{p}=.000$ con efecto bajo $(d=-.24)$. Siendo la media de los no repetidores superior a la media de los repetidores.

No se encontraron diferencias con la actitud del grupo de amigos, ni con la educación familiar en drogas así como en cuanto al estilo educativo.

Tabla 4

Factores de riesgo/ protección para el consumo de drogas en función de la repetición de curso

\begin{tabular}{lccc}
\hline \multicolumn{1}{c}{ Factores de riesgo/ protección } & & Repetidor & No repetidor \\
\hline \multirow{2}{*}{ Reacción de la familia ante el consumo } & $M$ & 28.07 & 26.10 \\
& $D . T$. & $(13.88)$ & $(10.15)$ \\
\hline \multirow{2}{*}{ Grupo de amigos } & $M$ & 19.76 & 19.74 \\
\hline \multirow{2}{*}{ Acceso a las drogas } & $D . T$. & $(6.64)$ & $(4.71)$ \\
\hline \multirow{2}{*}{ Riesgo familiar } & $M$ & 21.78 & 24.05 \\
& $D . T$. & $(10.94)$ & $(9.437)$ \\
\hline
\end{tabular}




\begin{tabular}{lccc}
\hline \multicolumn{1}{c}{ Factores de riesgo/ protección } & & Repetidor & No repetidor \\
\hline Educación familiar en drogas & $M$ & 16.20 & 16.31 \\
& $D . T$. & $(5.92)$ & $(4.82)$ \\
\hline \multirow{2}{*}{ Actividades protectoras } & $M$ & 45.60 & 48.50 \\
& $D . T$. & $(13.84)$ & $(8.79)$ \\
\hline \multirow{2}{*}{ Estilo educativo } & $M$ & 20.25 & 19.81 \\
& $D . T$. & $(9.26)$ & $(7.47)$ \\
\hline
\end{tabular}

Adaptación/Inadaptación integral y repetición de curso

La prueba t de Student, asumiendo varianzas heterogéneas, indicó la existencia de diferencias de medias significativas entre inadaptación escolar y repetición de curso $T(793.581)=3.022 ; \mathrm{p}=.003$ con efecto bajo $(d=.18)$. También en insatisfacción con el ambiente familiar $T(848.103)=2.240 ; \mathrm{p}=.025 \mathrm{con}$ efecto bajo $(d=.13)$. En ambos casos, la media de los repetidores fue superior a la media de los no repetidores (véase en la Tabla 5 las medias y desviaciones típicas de la prueba $t$ de Student para los alumnos repetidores y no repetidores en cada una de los factores de adaptación e inadaptación integral).

No se encontraron diferencias de medias significativas en la prueba t de Student en las variables inadaptación personal, inadaptación social, ni en insatisfacción con los hermanos, con respecto al hecho de haber repetido curso.

Tabla 5

Adaptación e inadaptación integral en función de la repetición de curso

\begin{tabular}{lccc}
\hline \multicolumn{1}{c}{ Adaptación/inadaptación } & & Repetidor & No repetidor \\
\hline \multirow{2}{*}{ Inadaptación general } & $M$ & 28.33 & 27.11 \\
& $D . T$. & $(15.79)$ & $(13.33)$ \\
\hline \multirow{2}{*}{ Inadaptación personal } & $M$ & 7.65 & 7.45 \\
& $D . T$. & $(5.98)$ & $(5.45)$ \\
\hline \multirow{2}{*}{ Inadaptación escolar } & $M$ & 12.36 & 11.06 \\
\hline \multirow{2}{*}{ Inadaptación social } & $D . T$. & $(7.73)$ & $(6.46)$ \\
\hline \multirow{2}{*}{ Insatisfacción con ambiente familiar } & $M$ & 8.33 & 8.60 \\
& $D . T$. & $(5.43)$ & $(4.79)$ \\
\hline \multirow{2}{*}{ Insatisfacción con los hermanos } & $M$ & .83 & .67 \\
& $D . T$. & $(1.24)$ & $(1.12)$ \\
\hline
\end{tabular}




\section{DISCUSIÓN Y CONCLUSIONES}

Los datos del estudio han permitido deducir que la repetición de curso puede ser explicada por variables sociodemográficas como: la titularidad del centro educativo, el sexo, la procedencia y la edad. Sin embargo, la ubicación geográfica del centro educativo, entre zona rural o urbana, no fue una variable significativa. De este modo, es destacable que los estudiantes que habían repetido curso solían pertenecer a centros educativos públicos; de sexo masculino; entre los alumnos extranjeros, más de la mitad habían repetido algún curso. Estos datos están en la línea de las investigaciones realizadas (Cordero et al., 2014; Lozano Díaz, 2009; Instituto Nacional de Evaluación Educativa, 2016; OCDE, 2016).

En lo referente al acoso escolar, la media de los agresivos que habían sido repetidores fue superior a la media de los estudiantes no repetidores; no siendo significativo en el caso de los alumnos víctimas (Cerezo, 1998, 2001, 2009; Pelegrín y Garcés de Los Fayos, 2009).

La preferencia social mostró que la media de los alumnos no repetidores fue superior a la media de los alumnos (no) repetidores. El impacto social no mostró diferencias entre los alumnos que habían repetido curso y los que no. El análisis del estatus reveló que entre los alumnos rechazados, aparece un mayor porcentaje de alumnos que han sido repetidores. Estos datos están en la línea de los efectos negativos de la repetición de curso apuntado por Farías et al. (2007) lo que puede exacerbar problemas de conducta y de ajustes socioemocionales (García-Bacete, et al., 2010).

De las variables situacionales del acoso escolar, desde un punto de vista global, los alumnos consideraban que el tipo de agresión más frecuente eran los insultos y amenazas, seguido del rechazo, maltrato físico y otras formas. Entre los estudiantes que presentaban un bajo rendimiento académico aparecía una mayor frecuencia y porcentaje en maltrato físico frente a los que poseían un rendimiento académico adecuado. En lo referente al lugar en el que ocurrían las agresiones, de forma global los estudiantes consideraban que las agresiones ocurrían sobre todo en el aula, seguido del patio, pasillos y otros sitios. Estos datos están en la línea con los estudios realizados que permiten concluir que los lugares donde suelen ocurrir las agresiones son el aula, el patio, los pasillos y otros lugares (Cerezo, 2009; Cerezo y Méndez, 2012). Entre los estudiantes repetidores, más de mitad consideraban que aparecían las agresiones en el aula.

En lo que respecta a la frecuencia de ocurrencia de las agresiones, los resultados mostraban que, independientemente de la repetición de curso, los alumnos consideraban que las agresiones solían ocurrir con una fre- 
cuencia de una o dos veces por semana, seguido de rara vez, todos los días y nunca. Estos resultados se fundamentan en los estudios previos que determinan que las agresiones entre los escolares ocurren con una frecuencia de una o dos veces por semana, rara vez, todos los día y nunca (Cerezo, 2009; Cerezo y Méndez, 2012).

En cuanto a la gravedad atribuida a las agresiones del acoso escolar, los resultados mostraban que la repetición de curso fue indiferente en la consideración del nivel de gravedad como: regular, seguido de poco o nada, bastante y mucho. Estos resultados se constatan en los resultados encontrados en estudios previos con dicho instrumento (Cerezo, 2009; Cerezo y Méndez, 2012).

$\mathrm{Al}$ analizar la percepción de seguridad/inseguridad en el centro escolar, los resultados mostraban que la mayor parte de los adolescentes que afirmaban sentirse bastante seguros en el centro escolar eran los que no habían repetido curso. Sin embargo, el mayor porcentaje de estudiantes repetidores percibían mucha seguridad en el centro escolar.

Estos datos sobre las variables situaciones así como la gravedad atribuida y la percepción de seguridad / inseguridad en el centro educativo pueden estar en la línea con el hecho de que los implicados en el acoso escolar como agresores también suelen ser repetidores de curso y por lo tanto son conocedores de las situaciones en las cuales participan (Cerezo, 1998, 2001, 2009; Pelegrín y Garcés de Los Fayos, 2009).

Los factores de riesgo para el consumo de drogas presentan cierta relación con la repetición de curso. Fue destacable que los estudiantes repetidores, consideraban que sus padres no se disgustarían ante un posible consumo de los hijos. Los no repetidores, sin embargo, destacaron una mayor accesibilidad a las drogas; alto riesgo familiar para el consumo de drogas por la existencia de consumo familiar en el hogar así como escasas actividades protectoras ante el consumo de drogas. Lo que puede ser indicativo de una mayor sensibilización ante el riesgo de consumo. Por otra parte, el haber repetido curso fue indiferente para la existencia de un grupo de amigos con consumo de drogas así como la educación familiar en drogas y el estilo educativo familiar. Los datos sobre los factores de riesgo o de protección para el consumo de drogas indican que los adolescentes, por lo general, se encuentran envueltos en actitudes de riesgo (Jessor, et al. 2003), lo cual podría llevarse a cabo independientemente de la repetición de curso.

Se encuentran elementos de inadaptación escolar e insatisfacción con el ambiente familiar relacionados con la repetición de curso, siendo por el contrario indiferente en cuanto a la inadaptación personal, inadaptación 
social, así como en insatisfacción con los hermanos. Algunos estudios encuentran que el bajo nivel de compromiso en las actividades académicas, el absentismo escolar, y el bajo rendimiento académico pueden ocasionar fracaso escolar e incluso la aparición de otras acciones de riesgo (Palacios y Andrade, 2007; Pinazo et al. 2002) ocasionando inadaptación escolar. Del mismo modo, entre los factores de riesgo familiar influye la estructura familiar (Cordero, et al., 2014) así como el clima de insatisfacción respecto al sistema familiar (Pons, 1998).

Entre las limitaciones del estudio cabría mencionar que algunas de las diferencias de medias encontradas son escasas, atendiendo al tamaño del efecto, por lo que sería adecuado haber tenido en cuenta otras variables tales como factores de personalidad, autoconcepto, motivación, así como contar con la perspectiva de los docentes y la familiar. Asimismo, se podría haber tenido en cuenta el número de cursos repetidos por el alumnado para poder precisar las diferencias encontradas en función del número de cursos repetidos. Por lo que, como prospectivas del estudio habría que superar las limitaciones encontradas e incluso plantear un estudio longitudinal de la repetición de curso para poder valorar el fracaso o abandono escolar producido.

Las conclusiones del estudio inciden en que es necesario llevar a cabo acciones de cooperación entre las familias y los centros educativos. Sería adecuado que los centros educativos promuevan actividades en las cuales las familias puedan colaborar de una forma estrecha con los docentes y orientadores así como con otras entidades comunitarias con la finalidad de lograr objetivos educativos rentabilizando esfuerzos y recursos. Asimismo, es necesaria la formación del profesorado en estrategias comunicativas y colaborativas con las familias, así como con otros agentes educativos y comunitarios (Martínez González y Álvarez Blanco, 2005). Asimismo, es necesario disponer de recursos que permitan detectar y abordar situaciones tales como el acoso escolar (Álvarez - García, et al. 2014), así como la presencia de otras conductas de riesgo de forma eficaz.

Finalmente este trabajo pone de relieve que se hace necesario llevar a cabo la prevención de la repetición del curso por parte del alumnado, evitando que presenten un desfase (Farías, et al., 2007) y por tanto problemas asociados como el consumo de drogas o la implicación en otras conductas problemáticas. 


\section{REFERENCIAS BIBLIOGRÁFICAS}

Alfonso, J. P., Huedo-Medina, T. B. y Espada, J. P. (2009). Factores de riesgo predictores del patrón de consumo de drogas durante la adolescencia. Anales de Psicología, 25(2) 330- 338.

Álvarez-García, D., Dobarro, A., Álvarez, L., Núñez, J. C. y Rodríguez, C. (2014). La violencia escolar en los centros de educación secundaria de Asturias desde la perspectiva del alumnado. Educación XX1, 17(2), 337-360. doi: 10.5944/educxx1.17.2.11494

Carabaña, J. (2013). Repetición de curso y puntuaciones PISA ¿Cuál causa cuál? En OCDE (Ed.), PISA 2012: Programa para la evaluación internacional de los alumnos. Informe español. Volumen II: Análisis secundario. (pp.33-66). Мadrid: Instituto Nacional de Evaluación Educativa. Subdirección General de Documentación y Publicaciones. Recuperado de https://goo.gl/IIs2qA

Carballo, J. L., García, O., Secades, R., Fernández, J. R., García, E., Errasti, J. M. y Al-Halabi, S. (2004). Construcción y validación de un cuestionario de factores de riesgo interpersonales para el consumo de drogas en la adolescencia. Psicothema, 16(4), 674-679.

Cerezo, F. (1998). Conductas agresivas en la edad escolar. Madrid: Pirámide

- (2001). La violencia en las aulas. Análisis y propuestas de intervención. Madrid: Pirámide

- (2009). Bullying: Análisis de la situación en las aulas españolas. International Journal of Psychology and Psychological Therapy, 9(3), 383-394.

- (2012). Bull-S. Test de evaluación de la agresividad entre escolares. Madrid/ Bizkaia: Albor-Cohs.

Cerezo, F. y Méndez, I. (2012). Conductas de riesgo social y de salud en ado- lescentes. Propuesta de intervención contextualizada para un caso de bullying. Anales de Psicología, 28(3), 705719. doi: 10.6018/analesps.28.3.156001

Cerezo, F. Méndez, I. y Ato, M. (2013). Moderating role of family and friends' factors between dissocial behaviour and consumption in adolescents. International Journal of Clinical and Health Psychology, 13(3), 171-180.

Cordero, J. M., Manchón, C. y Simancas, R. (2014). La repetición de curso y sus factores condicionantes en España. Revista de Educación, 365, 2-37. doi: 10.4438/1988-592X-RE-2014365-263

Delegación del Gobierno para el Plan Nacional sobre Drogas (DGPNSD) (2016). Informe 2016. Alcohol, tabaco y drogas ilegales en España. Madrid: Secretaría de Estado de Servicios Sociales e Igualdad. Recuperado des de https://goo.gl/oYJ5C3

Espada, J. P., Pereira, J. R. y García-Fernández, J. M. (2008). Influencia de los modelos sociales en el consumo de alcohol de los adolescentes. Psicothema, 20, 531-537.

Farías, M., Fiol, D., Kit, I. y Melgar, S. (2007). Todos pueden aprender - Propuestas para superar el fracaso escolar. Buenos Aires: Fondo de las Naciones Unidas para la infancia y Asociación civil educación para todos. Recuperado de https://goo.gl/ODsHhr

Garaigordobil, M. y Oñederra, J. A. (2010). La violencia entre iguales. Revisión teórica y estrategias de intervención. Madrid: Pirámide.

García- Bacete, F. J., Sureda, I. y Monjas, I. (2010). El rechazo entre iguales en la educación primaria: una panorámica general. Anales de Psicología, 26 (1), 123-136. 
Hernández-Guanir, P. (2015). TAMAI. Test autoevaluativo multifactorial de adaptación infantil. Madrid: TEA

Hymel, S. y Swearer S. M. (2015). Four decades of Research on School Bullying. An Introduction. American Psychological Association, 70(4), 293299 doi: 10.1037/a0038928

Inglés, C. J., Delgado, B., Bautista, R., Torregrosa, M., Espada, J., García-Fernández, J. M., Hidalgo, M. y García-López, L. (2007). Factores psicosociales relacionados con al consumo de alcohol y tabaco en adolescentes españoles. Internacional Journal of Clinical and Health Psychology, 7(2), 403-420.

Instituto Nacional de Evaluación Educativa (2016). Sistema Estatal de Indicadores de la Educación. Madrid: Secretaría General Técnica. Ministerio de Educación. Cultura y Deporte. Recuperado de https://goo.gl/UQQPjp

Jessor, R., Turbin, M. S., Costa, F. M., Dong, Q., Zhang., H. y Wang, Z. (2003). Adolescent problem behavior in China and United States: A crossnational study of psychosocial protective factors. Journal of Research on Adolescence, 13, 329-360.

Lozano Díaz, A. (2009). Factores personales, familiares y académicos que afectan al fracaso escolar en Educación Secundaria. Revista Electrónica de Investigación Psicoeducativa y Psicopedagógica, 1(1), 43-66.

Martínez González, R. A. y Álvarez Blanco, L. (2005). Fracaso y abandono escolar en Educación Secundaria Obligatoria: implicación de la familia y los centros escolares. Aula Abierta, 85, 127-146.

OCDE (2016). PISA 2015. Programa para la evaluación internacional de los alumnos. Informe español. Madrid: Instituto Nacional de Evaluación Educativa. Subdirección General de Documentación y Publicaciones. Recuperado de https://goo.gl/7tmAHP
Olweus, D. (1998). Conductas de acoso y amenaza entre escolares. Madrid: Morata.

Palacios, D. J. y Andrade, P. P. (2007). Desempeño académico y conductas de riesgo adolescente. Revista de educación y desarrollo, 7(6), 5-16.

Pelegrín, A. y Garcés de Los Fayos, E. J. (2009). Análisis de las variables que influyen en la adaptación y socialización: el comportamiento agresivo en la adolescencia. Ansiedad y estrés, 15(2-3), 131-150.

Pinazo, S., Pons, J. y Carreras, A. (2002). El consumo de inhalables y cannabis en la preadolescencia: Análisis multivariado de factores predisponentes. Anales de Psicología, 18(1), 77-93.

Pons, J. (1998). El modelado familiar y el papel educativo de los padres en la etiología del consumo de alcohol en los adolescentes. Revista Española Salud Pública, 72(3), 251-266.

Ruiz-Juan, F. y Ruiz-Risueño, J. (2011). Variables predictoras de consumo de alcohol entre adolescentes españoles. Anales de Psicología, 27(2), 350-359.

Secades, R., Carballo, J. L., FernándezHermida, J. R., García, O. y García, E. (2006). Cuestionario de factores de riesgo interpersonales para el consumo de drogas en adolescentes (FRIDA). Madrid: TEA.

Suárez, N., Tuero-Herrero, E., Bernardo, A., Fernández, E., Cerezo, R., González-Pienda, J. A., Rosario, P. y Núñez, J. C. (2011). El fracaso escolar en Educación Secundaria: Análisis del papel de la implicación familiar. Magister Revista miscelánea de investigación, 24, 49-64.

Sullivan, K., Cleary, M. y Sullivan, G. (2005). Bullying en la enseñanza secundaria. El acoso escolar: cómo se presenta y cómo afrontarlo. Barcelona: CEAC. 


\section{PERFIL ACADÉMICO Y PROFESIONAL DE LAS AUTORAS}

Inmaculada Méndez Mateo. Licenciada y Doctora en Psicología por la Universidad de Murcia. Profesora en el Departamento de Psicología Evolutiva y de la Educación de la Facultad de Psicología de la Universidad de Murcia. Los estudios llevados a cabo se han centrado en analizar las conductas de riesgo social y para la salud asociados a la problemática bullying; la formación del profesorado en el reconocimiento y detección del acoso escolar; la atención a la diversidad; los problemas de convivencia en alumnos con discapacidad intelectual, etc. Ha participado en diferentes jornadas, congresos y publicaciones científicas. Incluso ha realizado cursos de especialización y ha organizado congresos y jornadas de profesionalización.

Fuensanta Cerezo Ramírez. Profesora Titular de Psicología de la Educación de la Universidad de Murcia. Sus investigaciones están en la línea de la violencia escolar, conceptualización, evaluación, prevención e intervención; la adolescencia y sus problemas y formación del profesorado. Actualmente es la Investigadora Principal del grupo EIPSED-E042-01(Equipo de Investigación en Psicología de la Educación) de la Universidad de Murcia. Autora de numerosos estudios sobre violencia escolar, destacando el Test Bull-S para la evaluación sociométrica de la violencia escolar.

Dirección de las autoras: Inmaculada Méndez Mateo

Dpto. de Psicología Evolutiva

y de la Educación

Facultad de Psicología

Universidad de Murcia

Campus de Espinardo

30100 Murcia

E-mail: inmamendez@um.es

Fuensanta Cerezo Ramírez

Dpto. de Psicología Evolutiva

y de la Educación

Facultad de Psicología

Universidad de Murcia

Campus de Espinardo

30100 Murcia

E-mail: fcerezo@um.es

Fecha recepción del artículo: 13. Diciembre. 2014

Fecha modificación artículo: 24. Marzo. 2015

Fecha aceptación del artículo: 25. Abril. 2015

Fecha revisión para publicación: 31. Mayo. 2017 
